\title{
Implementing the Strengths Model of case management: Group supervision fidelity outcomes
}

Melissa Petrakis, Mick Wilson, Bridget Hamilton

Running title: Group supervision fidelity Strengths Model

Melissa Petrakis, Senior Research Fellow, $P h D, M S W$ (Research), $B S W, B A$

St Vincent's Mental Health Service and Lecturer, Department of Social Work, Faculty of Medicine,

Nursing and Health Sciences, Monash University

Mick Wilson, Manager, Footbridge Continuing Care Unit, RPN, P.G.Dip. Nursing Practice (Mental Health)

St Vincent's Mental Health Service

Bridget Hamilton, Senior Research Fellow, former Director of Nursing, NHMRC NICS Fellow, PhD, $B N($ Hon.), $R N$

St Vincent's Mental Health Service and Senior Lecturer, Department of Nursing, Melbourne School of Health Sciences, University of Melbourne

Dr Melissa Petrakis, Senior Research Fellow (Correspondence),

St Vincent's Mental Health Service, Hawthorn Community Mental Health Service, 642 Burwood Road, Hawthorn East 3123, Australia.

Phone: (03) 9882-9299 Fax: (03) 9882-9637

Email: Melissa.Petrakis@svhm.org.au 


\section{Introduction}

Evidence of clinical effectiveness is extremely important to key stakeholders in contemporary mental health service provision: clinicians, service managers, funding bodies, policy makers, and consumers and their families and carers. Evidence-based practice is increasingly prioritised and methods to achieve this are being developed (Aarons, Hurlburt, \& Horwitz, 2010; Benton, 1999; Chorpita, Daleiden, \& Weisz, 2005). Recent literature has noted the importance of 'empirically supported, theory-based treatments' and that 'intervention delivery must be evaluated for fidelity to content and process' (p. 2) to ensure outcomes can be accurately attributed to the intervention or its application (Schoenwald et al., 2010). The challenge though is that studies have shown that most consumers with severe and persistent mental illness frequently do not receive evidence-based therapeutic services (Farkas, Gagne, Anthony, \& Chamberlin, 2005; Lehman, Steinwachs, Dixon, Postrado, \& Scott, 1998; Torrey, Rapp, Van Tosh, McNabb, \& Ralph, 2005).

The difficulty at present is that, while consumer outcomes represent the best and most accurate measures of service effectiveness, accurate data is rarely available (Hamilton, 1999; Charles A. Rapp, Bond, Becker, Carpinello, \& Nikkel, 2005). Rapp and colleagues (2005, p. 354) suggest that 'In lieu of outcomes, states could reward the attainment of high fidelity', noting that, for example, New York used Assertive Community Treatment (ACT) fidelity measures to renew licenses, with longer-term licenses linked to higher fidelity scores. Measurements of fidelity quantify and track the core components of an evidence-based practice model or intervention and provide scales for ascertaining the degree of adherence. It 
has been noted that such measures can be used to establish clear standards, monitor meaningful performance of programs over time, improve performance, and document the relationship between adherence to a model and outcomes (Bond, Evans, Salyers, Williams, \& Kim, 2000; Charles A. Rapp, et al., 2005).

Strengths-based recovery models have become increasingly prevalent in mental health selfhelp agencies in the last 10 years (Hodges, Hardiman, \& Segal, 2004). A key goal that underlies this approach is to foster empowerment in individual service users (Dickerson, 1998). Though the recovery movement has gained momentum over the last twenty years, in the psychiatric disability rehabilitation and support sector, it is still only recently being heard and responded to in clinical psychiatric services both in Australia and overseas.

While few randomised controlled trial studies have investigated outcomes of case management based on the Strengths Model compared to standard care, initial results are promising (Björkman, Hansson, \& Sandlund, 2002). The Strengths Model has been found to be at least as effective as ACT on participant outcomes and utilization at 2 year follow-up (Barry, Zeber, Blow, \& Valenstein, 2003). Services that have moved towards high fidelity Strengths Model practice have reported outcomes such as increases in numbers of individuals living independently, numbers competitively employed, numbers involved in tertiary education, and decreases in psychiatric hospitalizations (Rapp \& Goscha 2006, p. 231).

Fidelity of Strengths Model implementation can be measured through use of the 12-item Strengths Case-Management Fidelity Scale (SCMFS). Two items explicitly relate to group supervision frequency and focus and a further four are impacted considerably by group supervision through the development of the strengths assessments, personal (goal) plans, the 
brainstorming of naturally occurring resources and an emphasis on hope-inducing behaviours (Rapp \& Goscha 2006, p. 264-6).

Group supervision for the authors of the model came about some 30 years ago to replace individual meetings as an efficiency measure (C.A. Rapp \& Chamberlain, 1985). Since then Rapp has attested that 'We have come to believe that group supervision is indispensable to effective case management practice' (Rapp and Goscha 2006, p. 218). In the Strengths Model, supervision sessions have three purposes: (1) support and affirmation of case manager efforts, ingenuity and accomplishments, (2) the generation of promising ideas to work more effectively with consumers, and (3) to facilitate case manager learning that can be applied to similar situations in future.

Brunero and Stein-Parbury (2008) noted (p. 87) that 'Whilst the practice of clinical supervision is established in other developed countries, such as the United Kingdom and the United States of America, at present it is underdeveloped in the Australian context'(Brunero \& Stein-Parbury, 2008). It has been observed though, in the United Kingdom, that many staff 'do not have regular, protected access to confidential conversations about the everyday challenges of their work, which would give them space and time to consider how they deal with increasing the quality of care with limited resources, and with ethical and moral issues' (Waskett, 2010a), further:

Often clinical supervision is "fudged" and slipped in under the headings of management supervision, appraisals, one to one meetings with team leaders, or even team meetings. 
How consumers view any model of practice implemented within area mental health services to develop and support recovery-oriented practices by case managers is very important. Qualitative investigations of strengths-based practice found that, while consumers experienced an initial mistrust of the approach and the challenge of holding on to strengths and deficits simultaneously, the approach was viewed favourably, especially the relationship with the case manager (Brun \& Rapp, 2001). From a consumer perspective, in a seminal paper more than 20 years ago, Patricia Deegan established four recommendations for creating programs that enhance recovery (Deegan, 1988). She noted (p.18) that: perhaps most fundamentally, staff attitudes are very important in shaping rehabilitation environments.

A review of forty years of outcome research in psychotherapy, including multidisciplinary perspectives, echoes this knowledge with the conclusion that the therapeutic relationship accounts for $30 \%$ of action or change achieved by clients (Duncan, Miller, \& Sparks, 2004).

The aim of this study is to evaluate the degree of fidelity to group supervision under the Strengths Model during its implementation in one public adult area mental health service; further to examine how this American model transfers and translates to an Australian clinical services context.

\section{Service development strategies}

In the Australian context, the Australian National Mental Health Plan 2003-2008 directed that services should adopt a recovery orientation (Commonwealth Government, 2003). During 2005-6 St Vincent's Mental Health Service (SVMH), Melbourne, determined to implement the Strengths Model as a development of existing case management practice (Chopra et al., 
2009). The model was directly and explicitly based on 'The Strengths Model: Case Management with people with psychiatric disabilities' (Rapp and Goscha, 2006). The purpose of adopting this model was to refocus case management offered by the service, in line with current evidence, mental health policy directions and in keeping with the values of the acute hospital.

The model was first implemented in the intensive rehabilitation services: the Mobile Support Team, and the residential Footbridge Continuing Care Unit (CCU). In 2007 the model was extended to Hawthorn and Clarendon clinics, the two community-based Continuing Care Teams (CCTs), providing case management to consumers with major mental illness.

The group supervision approach utilising Strengths Assessment and Goal Planning

\section{Figure 1}

Process description of Strengths group supervision at St Vincent's Mental Health

\section{Methods}

Strengths group supervision was initiated as a 3-month pilot (November 2008-January 2009 inclusive) across three sites: the intensive residential CCU and the two community CCT sites. This was conducted as described by Rapp and Goscha (2006). The supervision was held weekly at the CCU, for all but two weeks over Christmas, resulting in 10 sessions. For the two community-based CCT sites supervision was held fortnightly, resulting in 6 sessions for one site, and 6 and 5 sessions for the two teams at the second site.

The group supervision feedback documents as tools in evaluation 
For the pilot the supervision process was monitored through the use of three documents: the facilitator's feedback form, the presenter's feedback form and multiple copies of the group feedback form completed for each session. The facilitator's feedback form was directly modelled on the questions for 'Supervisor's Group Supervision Monitor', pages 233-4 in the Rapp and Goscha text (C.A. Rapp \& Goscha, 2006). The presenter's feedback form was directly modelled on the questions for 'Case Managers - Group Supervision Feedback', pages 232-3 (C.A. Rapp \& Goscha, 2006). All group participants also completed a feedback form based on the 'Case Managers - Group Supervision Feedback', to assist them to orient themselves to the Strengths process and remain actively engaged with the monitoring of strengths-sensitive practice, even when they were not themselves presenting a case. These group feedback forms served as a reiteration and cross-check of the presenter's feedback form. The forms were standard, however it should be noted that CCT site 1 utilised twothirds of the facilitator feedback items rather than the full complement adopted at the other two sites for the pilot. In analysis for the seven missing items the mean was substituted.

\section{Results}

Twenty-five of the 26 items set out by Rapp and Goscha (2006, p. 233-4) for the 'Supervisor's Group Supervision Monitor' were audited (Table 1). The final item 'Group identified policies requiring advocacy' was omitted for the pilot since policy redevelopment was beyond the scope of the initial piloting of these tools. These were sub-grouped into 'Group Interaction’ and 'Client Work' as suggested by Rapp and Goscha (2006). Fidelity audit data from the intensive residential rehabilitation CCU unit and the two community CCT sites, including two teams within one site was collected.

\section{Table 1}

'Supervisor's Group Supervision Monitor' for Facilitator Feedback 
High fidelity was achieved for Group Interaction. The mean fidelity score was 20.2 (74.8\%). Fidelity was most strong for 'People left feeling energised/more optimistic,' for 'Seating circular and comfortable' and 'Discussion among all participants' (Figure 2). There was lower fidelity for 'No interruptions,' for 'No person made excuses to shoot down potential resources or ideas' and 'Conscientious efforts which failed celebrated'.

\section{Figure 2}

\section{Group Interaction fidelity}

High fidelity was achieved for Client Work. The mean fidelity score was 20.8 (77\%). Fidelity was most strong for 'Three or more options or ideas for tasks forward,' for 'Discussion ends with a specific plan or strategy' and 'Discussion ends with specific tasks to be done' (Figure 3). There was lower fidelity for 'Patterns or similarities between situations identified,' for 'Did reframe problems/deficits to interests/strengths or goals' and 'Group members received positive feedback regarding the use of natural helpers'.

\section{Figure 3}

\section{Client Work fidelity}

All 7 items, including sub-items, set out by Rapp and Goscha (2006, p. 232-3) for the 'Case Managers - Group Supervision Feedback' were audited (Table 2). The form within our service has been titled the Presenter Feedback form.

\section{Table 2}

'Case Managers - Group Supervision Feedback' for Presenter Feedback 
High fidelity was achieved for Client Work. The mean fidelity score was 24.3 (90\%). Fidelity was most strong for 'Receive help to: Identify goals, tasks or strategies,' for 'Receive help to: Identify natural occurring non-mental health resources,' for 'Receive help to: Identify client strengths,' and both ' 3 or more options or ideas for tasks forward' and identification of specific 'Positive feedback on.../Successes identified...' (Figure 4). There was lower fidelity only for 'Conscientious efforts which failed acknowledged'.

\section{Figure 4}

\section{Presenter fidelity}

\section{Qualitative feedback}

Following the 3-month pilot feedback was sought from staff who wished to clarify what was working well or could be improved. Positive feedback received emphasised that Strengths group supervision re-invigorated practice. There were slight differences in tone based on level of professional experience that the supervision process was contributing to. As one occupational therapist noted: "as a clinician you have a refreshed view and some new ideas which you can bring to the client." As one graduate nurse noted:

Generally I have found the Strengths supervision to be useful. The brainstorming sessions provide useful ideas for the clinician presenting a consumer as well as other clinicians who may take ideas to other consumers. I also feel it helps clinicians to get some perspective on the challenges they may be encountering with the consumer they care for. Also it is very encouraging to here all clinicians express positive experiences they have had with clients.

Critical feedback concerned process aspects to be addressed following the pilot, prior to service-wide implementation. As one graduate nurse noted: 
A problem I find with strengths supervision is that the discussion prior to brainstorming can be unfocused and the purpose of this section unclear if the clinician running the session does not actively manage the group.

In suggesting recommendations, one experienced psychiatric nurse noted: "I think it could be useful in the future to consider the client being involved in the supervision session, obviously depending on mental state and level of engagement."

\section{Discussion}

Internationally there have been considerable challenges noted in implementing evidencebased practices into mental health services (Lehman, et al., 1998). Changing mental health professional practice to recovery-oriented principles has met resistance, even when there has been a highly supportive policy environment, strong management support, and positive staff response following training (Deane, Crowe, King, Kavanagh, \& Oades, 2006).

The results of this study indicate that St Vincent's Mental Health Service has been faithful in its implementation of the Strengths Model of Case Management as outlined by Rapp and Goscha (2006). There has been consistent and careful application of process measures and paperwork to facilitate case managers becoming strengths-oriented in their practice. When case managers present how they are working with consumers and what they need support and assistance to achieve in a still more strengths oriented manner, this was viewed by clinicians as a highly productive undertaking. This is evidenced by the presenter feedback result of $90 \%$ fidelity to group supervision according to this model. There has been consistent application of 
approach by those senior clinicians facilitating group brainstorming to support case manager efforts in undertaking meaningful and strengths-focused reflections, and the planning of their ongoing casework.

It is important to note that there has been a language shift in our implementation at $\mathrm{St}$ Vincent's Mental Health from 'Supervisor' to 'Facilitator'. The supervision has been uncoupled from line or performance management and instead is facilitated by an interested Senior Clinician peer. Further, this monitoring - initially called ‘Group Supervision’ as per Rapp and Goscha (2006, p. 216-234) - is now called 'Strengths Brainstorming'. Again, staff feedback was that it was easier to be consumer-centred, goal-oriented and solution-focused in a session that was not conceptualised as 'supervision'. Since the key purpose of the meetings are to brainstorm consumer goals as a team, to open up new ideas and creative solutions to take back to the consumer, 'brainstorming' seemed a more appropriate term (Osborn, 1963; Rickards, 1999).

While these three tools were designed to facilitate supporting clinicians to adopt consumercentred and strengths-oriented practice, they proved to be meaningful documents in an audit sense. They were efficacious as evaluative tools to monitor program and service adherence to the group supervision component of the Strengths Model. It has been noted that 'Supervision is likely to be established in a more sustainable way if the whole organisational process is carefully designed and monitored' (Hawkins \& Shohet, 2003). The importance of baseline measurement taken at an early stage for later comparison has been emphasised (Waskett, 2010b). In the results the facilitator and presenter feedback were collated, to clarify positive achievements in staff use of group supervision as well as areas for further development; a 
process in keeping with, or a mirroring of, the reflective and respectful framing that is a hallmark of this strengths-oriented work with consumers.

It is possible that there are some cultural differences between a North American communitybased care context and an Australian clinical mental health service context. It is not immediately apparent to our staff, for example, that laughter in a group brainstorming session is a good thing or strengths-oriented. Many case managers and facilitators are understandably earnest in their attempts to use brainstorming to gain ideas to best support the consumers they work with. A conservative view of the appropriateness of laughter has negatively impacted fidelity scores on items 4 and 5 for CCT site 1 in the 'Group Interaction' section on the facilitator feedback form and item 14 for the CCU on the presenter feedback form.

For all four sites or teams there was low fidelity for the item of 'no interruptions,' at item 7 in the 'Group Interaction' section on the facilitator feedback form. This goal may not be readily achievable in a clinical context where crisis presentations for consumers are not uncommon. A low score may even be indicative of prioritising flexibility in meeting consumer needs and goals ahead of service quality assurance activities, rather than implying a lack of strengthsfocused work being undertaken.

There were some site or team differences in interpretation of certain items. An example is that in facilitator feedback for 'Group Interaction' item 10, at the intensive residential CCU service, ideas were 'generated by the majority of the group' 9 out of 10 sessions. For CCT site 1 this was the case in 2 out of 6 of sessions, however the response to item 9 noted that simultaneously 'an individual did not dominate the process' in 6 out of 6 sessions. A very low item 9 score of fidelity for 1 out of 5 sessions from one team at CCT site 2, despite an 
overall group interaction fidelity mean of 4 out of 5 , is further indication that these items taken individually may not reflect the fidelity being achieved. Dominance of a group member may not infer how strengths-oriented contributions are. Most groups have norms that become established over time, including that certain individuals may be looked to as leaders, with contributions particularly valued or beneficial to the group, and assertiveness by others may also be tolerated and absorbed.

That said, the fidelity items taken as a group were useful to assist a service, program or team in becoming strengths-focused, particularly during the establishment phase of utilising the Strengths Model. Totals for supervisor fidelity to group interaction and client work, and the extent to which the case manager presenting felt they received the help they sought, were highly relevant to fidelity of a Strengths Model as implementation was taking place, to monitor and modify that implementation. The items were useful prompts, enabling measurement and auditing, to ensure teams and programs remained faithfully strengthsoriented in their approach to reflecting on the work they were undertaking with the consumers they were aiming to support and assist.

\section{Limitations}

Although some diversity of case management and rehabilitation contexts was incorporated, with both continuing care and residential services included, a limitation of the study was that as all three sites were from the same mental health service. It may not be possible to generalise results to other programs and services with their own specific organisational cultures, practices and policies. There may have been variations in staffing, the implementation of protocols, and program emphasis at each site that can affect outcomes 
(Barry, et al., 2003). The study was one based in a clinical service setting, and in an Australian context, so results and implications may not be directly transferrable to psychiatric disability rehabilitation and support service contexts and to other programs and service cultures.

It should not be underestimated that St Vincent's Hospital Melbourne is explicitly a valuesbased organisation, and one that comes with a long history of ministering to those challenged by adversity in addition to ill health. The mission of the Sisters of Charity is guided by the values of compassion, justice, human dignity, excellence and unity; values that informed the Mental Health Service in its substantive implementation of a recovery approach and its endeavours to bring this in systematically across all components of the service. It may not be readily possible to implement the group supervision aspects of the Strengths Model as outlined by Rapp and Goscha (2006) in other clinical services without local modifications.

\section{Conclusions}

This evaluation of fidelity in the group supervision component of the Strengths Model of case management, implemented within one area mental health service, suggests that the two sets of items provided by Rapp and Goscha (2006) to confirm fidelity have utility for group facilitators and case managers. The group supervision feedback items for case managers were directly applicable when presenting challenges they were seeking to address in their work with consumers. The supervisor's group supervision monitor was beneficial during implementation of the model, however somewhat long at 26 items for ongoing use. It was 
unclear whether items on laughter in sessions and the extent to which ideas were generated by all group members were reliably reflective of the Strengths Model in use.

\section{Acknowledgements}

We thank our colleagues for their input during the implementation of group supervision, members of the St Vincent's Mental Health Service Strengths Model Implementation Group. Thanks to Tony Pinzone for assisting with design of the database for monitoring group supervision. Thanks to David Castle and Bella Anderson for reviewing an earlier version of this paper. Thank you to the clients, clinicians and management of St Vincent's Mental Health Service. This research was conducted through the allocation of clinical time and management support over the 3 month pilot period, and a 0.2 EFT researcher position over a later 3 months to analyse data collected. The preparation of this paper for publication was supported by the Victorian Department of Health 'Health System and Practice Improvement Grants - positive culture and leadership grant'.

\section{References}

Aarons, G. A., Hurlburt, M., \& Horwitz, S. M. (2010). Advancing a conceptual model of evidence-based practice implementation in public service sectors. Administration and Policy in Mental Health and Mental Health Services Research.

Barry, K. L., Zeber, J. E., Blow, F. C., \& Valenstein, M. (2003). Effect of Strengths Model versus Assertive Community Treatment Model on Participant Outcomes and Utilization: Two-Year Follow-Up. Psychiatric Rehabilitation Journal, 26(3), 268278.

Benton, D. C. (1999). Clinical effectiveness. In S. Hamer \& G. Collinson (Eds.), Achieving Evidence-Based Practice: A Handbook for Practitioners. London: Balliere Tindall, an imprint of Harcourt Publishers Limited.

Björkman, T., Hansson, L., \& Sandlund, M. (2002). Outcome of case management based on the strengths model compared to standard care. A randomised controlled trial. Social Psychiatry and Psychiatric Epidemiology, 37(4), 147-152.

Bond, G. R., Evans, L., Salyers, J., Williams, M. P., \& Kim, H. (2000). Measurement of fidelity in psychiatric rehabilitation. Mental Health Services Research, 2(2), 75-87. 
Brun, C., \& Rapp, R. C. (2001). Strengths-Based Case Management: Individuals' Perspectives on Strengths and the Case Manager Relationship. Social Work, 46(3), 278-288.

Brunero, S., \& Stein-Parbury, J. (2008). The effectiveness of clinical supervision in nursing: an evidence based literature review. Australian Journal of Advanced Nursing, 25(3), 86-94.

Chopra, P., Hamilton, B., Castle, D., Smith, J., Mileshkin, C., Deans, M., et al. (2009). Implementation of the Strengths Model at an area mental health service. Australasian Psychiatry, 17(3), 202-206.

Chorpita, B. F., Daleiden, E. L., \& Weisz, J. R. (2005). Identifying and selecting the common elements of evidence based interventions: A distillation and matching model. Mental Health Services Research, 7, 5-20.

Commonwealth Government. (2003). National Mental Health Plan 2003-2008. Canberra: Australian Government Publishing Service.

Deane, F. P., Crowe, T. P., King, R., Kavanagh, D. J., \& Oades, L. G. (2006). Challenges in implementing evidence- based practices into mental health services. Australian Health Review, 30(3), 305-309.

Deegan, P. E. (1988). Recovery: The lived experience of rehabilitation. Psychosocial Rehabilitation Journal 11(4), 11-19.

Dickerson, F. B. (1998). Strategies that foster empowerment. Cognitive and Behavioral Practice, 5(2), 255-275.

Duncan, B. L., Miller, S. D., \& Sparks, J. A. (2004). The Heroic Client: A Revolutionary Way to Improve Effectiveness Through Client-Directed, Outcome-Informed Therapy. San Francisco, CA: John Wiley \& Sons, Inc. .

Farkas, M., Gagne, C., Anthony, W., \& Chamberlin, J. (2005). Implementing recovery oriented evidence based programs: identifying the critical dimensions. Community Mental Health, 41(2), 141-158.

Hamilton, J. (1999). Quality according to QISMC. In K. Coughlin (Ed.), Medicaid managed behavioral care sourcebook: Strategies and opportunities for providers and purchasers (pp. 368-370). New York: Faulkner \& Gray.

Hawkins, P., \& Shohet, R. (2003). Supervsion in the Helping Professions. Maidenhead: Open University Press.

Hodges, J. Q., Hardiman, E. R., \& Segal, S. P. (2004). Predictors of hope among members of mental health self-help agencies. Social Work in Mental Health, 2(1), 1-16.

Lehman, A. F., Steinwachs, D. M., Dixon, L. B., Postrado, L., \& Scott, J. E. (1998). Patterns of usual care for schizophrenia: Initial results from the Schizophrenia Patient Outcomes Research Team (PORT) Client Survey. Schizophrenia Bulletin, 24(1), 1123.

Osborn, A. F. (1963). Applied imagination: Principles and procedures of creative problem solving (3rd revised ed.). New York, NY: Charles Scribner's Sons.

Rapp, C. A., Bond, G. R., Becker, D. R., Carpinello, S. E., \& Nikkel, R. E. e. a. (2005). The Role of State Mental Health Authorities in Promoting Improved Client Outcomes through Evidence-Based Practice. Community Mental Health Journal, 41(3), 347-363.

Rapp, C. A., \& Chamberlain, R. (1985). Case managment services to the chronically mentally ill. Social Work, 30(5), 417-422.

Rapp, C. A., \& Goscha, R. J. (2006). The Strengths Model: Case Management with People with Psychiatric Disabilities (Second ed.). New York: Oxford University Press.

Rickards, T. (1999). Brainstorming. In M. Runco \& S. Pritzker (Eds.), Encyclopedia of Creativity (Vol. 1, pp. 219-228). San Diego: Academic Press. 
Schoenwald, S. K., Garland, A. F., Chapman, J. E., Frazier, S. L., Sheidow, A. J., \& Southam-Gerow, M. A. (2010). Toward the Effective and Efficient Measurement of Implementation Fidelity. Adm Policy Ment Health.

Torrey, W. C., Rapp, C. A., Van Tosh, L., McNabb, C. R. A., \& Ralph, R. O. (2005).

Recovery principles and evidence-based practice: essential ingredients of service improvement. Community Mental Health, 41(1), 91-100.

Waskett, C. (2010a). Clinical supervision using the 4S model 1: considering the structure and setting it up Nursing Times, 106(16).

Waskett, C. (2010b). Clinical supervision using the 4S model 3: how to support supervisors and sustain schemes. Nursing Times, 106(17).

\title{
Table 1
}

\section{'Supervisor's Group Supervision Monitor' for Facilitator Feedback}

\author{
Group Interaction \\ 1. Session start on time \\ 2. Seating circular and comfortable \\ 3. Discussion among all participants \\ 4. Own laughter in session \\ 5. Participants laughter in session \\ 6. Atmosphere optimistic and positive \\ 7. No interruptions \\ 8. Client situations were reviewed \\ 9. Individual did not dominate the process \\ 10. Ideas generated by majority of the group \\ 11. No person made excuses to shoot down potential resources or ideas \\ 12. Conscientious efforts which failed celebrated \\ 13. People left feeling energised/more optimistic
}

\section{Client Work}

1. Three or more options or ideas for tasks forward

2. Discussion ends with a specific plan or strategy

3. Discussion ends with specific tasks to be done

4. If frustrations help the clinician reframe expectations and/or break tasks into smaller steps

5. Use of the strengths assessment to identify goals, task or strategies

6. Community resources identified

7. Strategies for involving natural helpers

8. Did reframe 'problems'/'deficits' to 'interests'/'strengths' or 'goals'

9. Patterns or similarities between situations identified

10. Group identified and celebrated successes

11. Group members received positive feedback regarding the use of client strengths

12. Group members received positive feedback regarding the use of natural helpers

13. Group identified policies requiring advocacy

Table 2

'Case Managers - Group Supervision Feedback' for Presenter Feedback

\section{Receive help to:}

1. Identify goals, tasks or strategies

2. Identify natural occurring non-mental health resources

3. Identify activities to be done by client/community

4. Break goals into smaller tasks 
5. Translating problems into goals

6. Identify patterns or similarities between cases

7. Identify what clients want to work on

8. Engage clients in relationship

9. Identify client strengths

10. 3 or more options or ideas for tasks forward

11. Atmosphere optimistic and positive

12. Positive feedback on.../Successes identified...

13. Conscientious efforts which failed acknowledged

14. Own laughter/enjoyment in session

\section{Figure 1}

Process description of Strengths group supervision at St Vincent's Mental Health

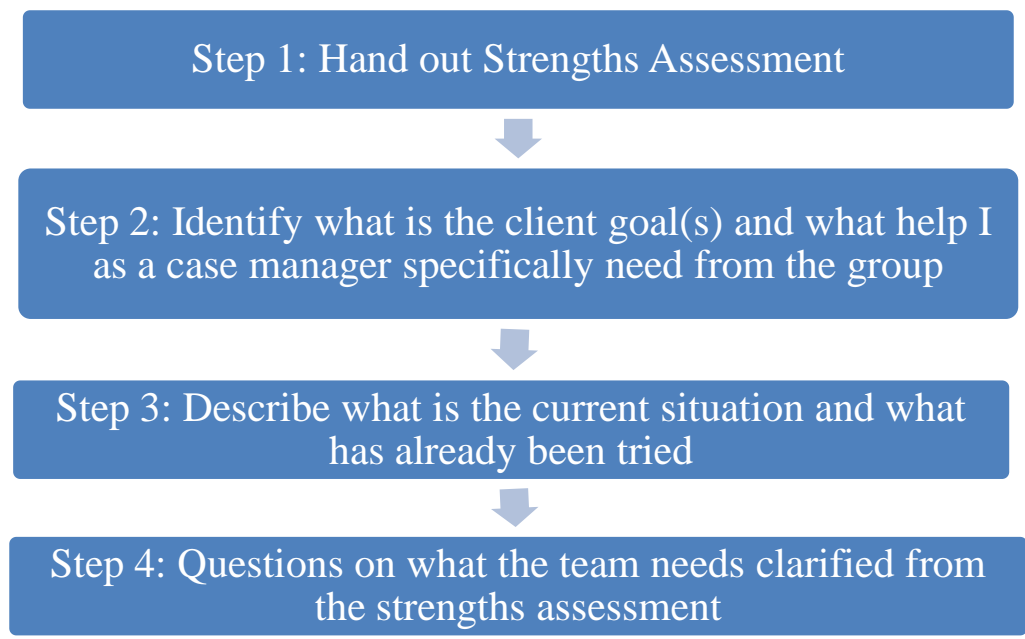

Step 5: Brainstorming ideas from the group for enhancing how the case manager works with the client goals (10-15 mins)

Step 6: Determine what will be my plan based upon the suggestions made to take back to the client

\section{Figure 2}

Group Interaction fidelity 


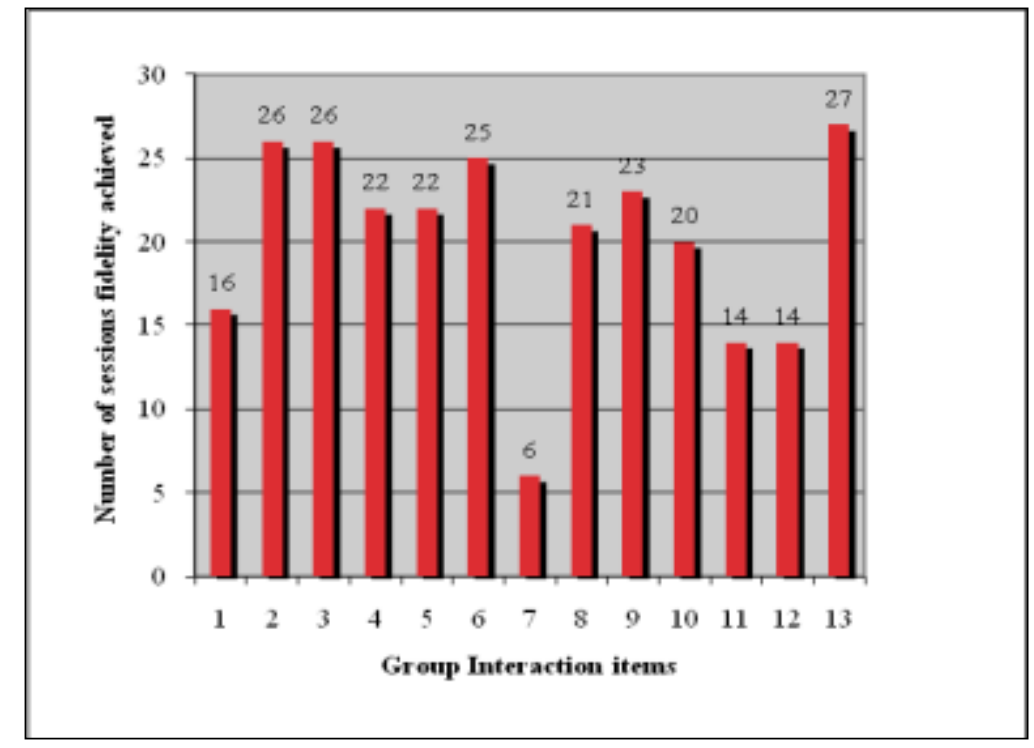

Figure 3

Client Work fidelity

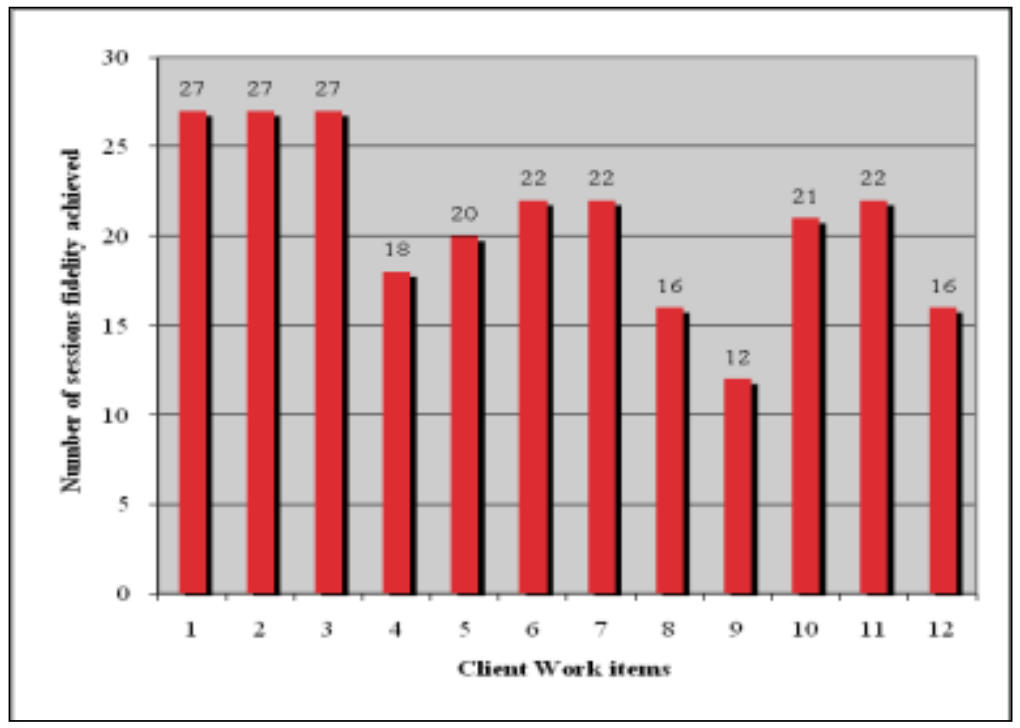

Figure 4

Presenter fidelity 


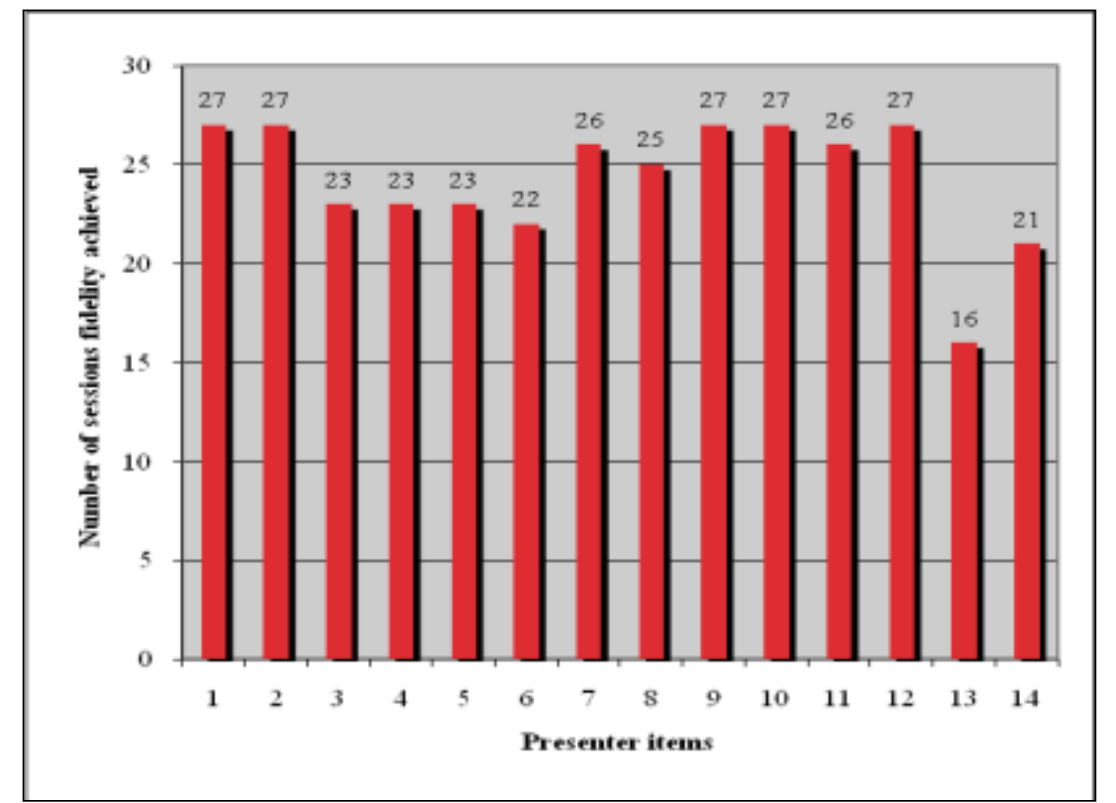

